\title{
Fréchet Distance Problems in Weighted Regions *
}

\author{
Yam Ki Cheung and Ovidiu Daescu \\ Department of Computer Science, \\ University of Texas at Dallas, \\ Richardson, TX 75080, USA, \\ \{ykcheung, daescu\}@utdallas.edu
}

\begin{abstract}
We discuss two versions of the Fréchet distance problem in weighted planar subdivisions. In the first one, the distance between two points is the weighted length of the line segment joining the points. In the second one, the distance between two points is the length of the shortest path between the points. In both cases, we give algorithms for finding a $(1+\epsilon)$-factor approximation of the Fréchet distance between two polygonal curves. We also consider the Fréchet distance between two polygonal curves among polyhedral obstacles in $\mathcal{R}^{3}(1 / \infty$ weighted region problem) and present a $(1+\epsilon)$-factor approximation algorithm.
\end{abstract}

\section{Introduction}

Measuring similarity between curves is a fundamental problem that appears in various applications, including computer graphics and computer vision, pattern recognition, robotics, and structural biology. One common choice for measuring the similarity between curves is the Fréchet distance, introduced by Fréchet in 1906 [16]. The traditional (continuous) Fréchet distance $\delta_{F}$ for two parametric curves $P, Q:[0,1] \rightarrow \mathcal{R}^{d}$ is defined as

$$
\delta_{F}(P, Q)=\inf _{\alpha, \beta:[0,1] \rightarrow[0,1]} \sup _{r \in[0,1]} S(P(\alpha(r)), Q(\beta(r))),
$$

where $\alpha$ and $\beta$ range over all continuous non-decreasing functions with $\alpha(0)=\beta(0)=0$ and $\alpha(1)=\beta(1)=1, S$ is a distance metric between points, and $d>0$ is the dimension of the problem.

The Fréchet distance is described intuitively by a man walking a dog on a leash. The man follows a curve (path), and the dog

\footnotetext{
* This work was supported in part by NSF award CCF-0635013
} 


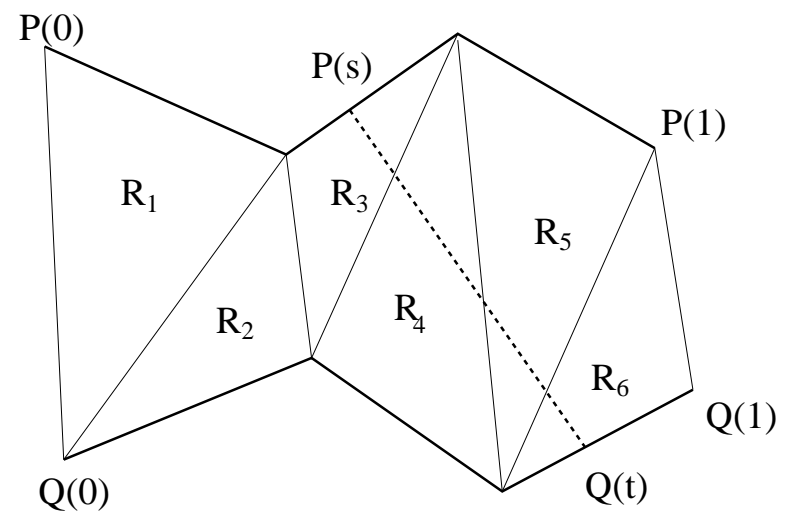

Fig. 1. An example of the Fréchet distance problem in weighted regions.

follows the other. Both can control their speed independently, but backtracking is not allowed. The Fréchet distance between the curves is the length of the shortest leash that is sufficient for the man and the dog to walk their paths from start to end.

A reparameterization is a continuous, non-decreasing surjective function. The pair $\alpha, \beta$ of reparametrizations define how the end points of the leash, i.e. $P(\alpha(r))$ and $Q(\beta(r))$, sweep along their respective curves. We say that $\alpha, \beta$ is a matching between $P$ and $Q$ or $\alpha, \beta$ define a monotone walk from leash $P(0) Q(0)$ to leash $P(1) Q(1)$. In this We use $s$ and $t$ to denote $\alpha(r)$ and $\beta(r)$, respectively. We assume $P$ and $Q$ are polygonal, and denote by $\delta(P)=\left(u_{1}, u_{2}, \ldots, u_{p}\right)$ and $\delta(Q)=\left(v_{1}, v_{2}, \ldots, v_{q}\right)$ the sequences of vertices of $P$ and $Q$, respectively.

In this paper, we study the Fréchet distance in weighted regions. In this problem, a $2 D$ plane is divided into a subdivision $R=\left\{R_{1}, R_{2}, \ldots, R_{n^{\prime}}\right\}$ with a total of $n$ vertices, with each region $R_{i} \in R$ having associated a positive integer weight $w_{i}$. The length of a path $\pi$ that stays within a region $R_{i}$ of $R$ is defined as $w_{i}|\pi|$, where $|\pi|$ is the Euclidean length of $\pi$. The length of a path in $R$ is the sum of the lengths of the subpaths within each region of $R$.

Let $P(s) Q(t)$ be the leash with endpoints $P(s)$ and $Q(t)$. Let $R_{i}(P(s) Q(t))$ be the Euclidean length of the line segment with endpoints $P(s)$ and $Q(t)$ within the region $R_{i}$. We define the distance between two points $P(s)$ and $Q(t)$ on $P$ and $Q$, respectively, as (a) 
$S(P(s), Q(t))=\sum_{i=1}^{n^{\prime}} w_{i} * R_{i}(P(s) Q(t))$ or (b) $S(P(s), Q(t))=$ the length of the shortest (weighted) path from $P(s)$ to $Q(t)$.

We formally define the Fréchet distance problem in weighted regions as the following: Given a $2 D$ weighted subdivision $R$ and two parameterized polygonal chains $P$ and $Q$ in $R$, find the Fréchet distance between $P$ and $Q$, where the distance between two points $P(s)$ and $Q(t)$ on $P$ and $Q$, respectively, is defined either as in (a) or as in (b) above.

Without loss of generality, we assume $R$ is triangulated and $P$ and $Q$ lie on boundaries of $R$ (see Fig. 1 as an illustration). We also discuss a special case in $\mathcal{R}^{3}$, where the weights are either 1 or $\infty$ (i.e., free space and obstacles).

\subsection{Motivation}

The motivation for studying such measurement comes from path/travel planning. Let us consider the following scenario. Suppose during a military operation, there are two teams of military units traveling on separate paths. However, before reaching their own destinations, the two teams want to maintain constant radio communications so that in case of any emergency one team can rescue the other team in time. The question is how should these two teams schedule their trips such that at any given time, the radio signal received by one team from the other is stronger than a threshold, or what is the optimal traveling schedule for each team such that minimum signal strength received is maximized.

We use the Beer-Lambert law to model the decay (of the intensity) of an EM wave traveling through a region. Beer-Lambert law states that there is a logarithmic dependence between the transmission $T$ of the EM wave and the product of the absorption coefficient associated with the region $\alpha$ and the distance $l$ traveled by the EM wave in the region, i.e. $T=\frac{I}{I_{o}}=e^{-\alpha l}$, where $I_{o}$ and $I$ are the initial intensity (or power) of the EM wave and the intensity after the path, respectively [17]. If the EM wave travels through a set of regions $R=\left\{R_{1}, R_{2}, \ldots, R_{k}\right\}$, the transmission $T$ can be written as $T=e^{\sum_{i=1}^{k} \alpha_{i} l_{i}}$, where $\alpha_{i}$ and $l_{i}$ are the absorption coefficient associated with $R_{i}$ and the distance traveled by the EM wave in $R_{i}$, respectively. The decay of the wave intensity can also be expressed in terms 
of the absorbance $A$ which is defined as $A=-\log _{10} \frac{I}{I_{0}}=\sum_{i=1}^{k} \alpha_{i} l_{i}$. Notice that if we treat $R$ as a weighted subdivision, where the weight of each region $R_{i}$ is its absorption coefficient $\alpha_{i}, A$ is exactly the weighted length of the path traveled by the EM wave. Hence, this scheduling problem can be reduced to the Fréchet distance problem in weighted regions.

\subsection{Previous Work}

The Fréchet distance and its variants attracted considerable attention in literature. Most previous work assumes an unweighted environment and can be divided into two categories, depending on the distance metric used. In the first category, the distance between two points is the Euclidean distance. In other words, the leash is always a line segment, as in case (a) above. Bending of the leash is not allowed. Fréchet distances in this category are also referred to as non-geodesic Fréchet distances.

Although Fréchet distance was introduced in 1906, it was not until 1993 that Alt and Godau [4] gave the first polynomial-time algorithm that computes the exact non-geodesic Fréchet distance between two polygonal curves $P$ and $Q$. They first gave an $O(p q)$ time algorithm for solving the decision version of the problem, which is the problem to decide, for a given constant $\Delta$, whether the Fréchet distance between two curves is at most $\Delta$. Then, they showed how to solved optimization version of the problem, which is the problem to find the exact Fréchet distance, in $O(p q \log p q)$ time by applying the decision algorithm and parametric searching technique. They also presented a more practical alternative solution, which takes $O\left(\left(p^{2} q+\right.\right.$ $\left.\left.q^{2} p\right) \log (p q)\right)$ time, by running binary search over a set of critical values.

Shortly after, Either and Mannila [15] introduced the concept of the discrete Fréchet distance $\delta_{d F}$, which considers only order preserving pairing of vertices in the polygonal curves. They gave an $O(p q)$ time algorithm for computing the discrete Fréchet distance. They showed that the difference between the discrete Fréchet distance and exact Fréchet distance between two curves is at most the length of the longest edge of the curves. As a result, discrete Fréchet distance can be can be used for approximation of the exact Fréchet 
distance between two arbitrary curves. The approximation error decreases as more vertices (Steiner pionts) are introduced to the two curves.

Rote [20] explored the Fréchet distance between more general curves. He gave an $O(p q \log p q)$ time algorithms for finding the Fréchet distance between piecewise smooth curves. The decision version of the problem can be answered in $O(p q)$ time. Wang et al. [7] studied the problem of measuring partial similarity between curves. They presented the first exact polynomial-time algorithm to compute a partial matching between two polygonal curves, which maximizes the total length of subcurves of them that are similar to each other, where similarity is measured in Fréchet distance under $L_{1}$ or $L_{\infty}$ norm. Their algorithm takes $O\left(p q(p+q)^{2} \log (p q)\right)$ time. Buchin et al. [6] studied the Fréechet distance between simple polygons and showed that it can be computed in polynomial time. They gave the first polynomial-time algorithm for computing the Fréchet distance between simple polygons.

In the second category, the distance between two points is the geodesic distance. Fréchet distances in this category are also referred to as geodesic Fréchet distances. The leash is allowed to bend to achieve to the minimum length. Maheshwari and Yi [18] study the case in which the curves lie on a convex polyhedron. By constructing a visibility diagram that encodes shortest path information for any pair of points on curves, one from each curve, they gave a $O\left(\left(p^{2} q+\right.\right.$ $\left.\left.p q^{2}\right) k^{4} \log (p q k)\right)$ time algorithm, where $k$ is the number of faces of the polyhedron. Cook and Wenk [14] gave a $O\left(k+M^{2} \log (k M) \log M\right)$ expected time or $O\left(k+M^{3} \log (k M)\right)$ worst-case time algorithm for computing the Fréchet distance between two polygonal curves when the leash is constrained inside a polygon, where $k$ complexity of the polygon and $M$ is the larger of the complexities of the two curves. Chambers et al. [8] showed how to compute the homotopic Fréchet distance between two polygonal curves in the presence of obstacles in polynomial time. The homotopic Fréchet distance is the Fréchet distance with an additional continuity requirement: the leash is not allowed to switch discontinuously, e.g. jump over obstacles is forbidden unless the leash is long enough.

Weighted region shortest path problems have been investigated in computational geometry for about two decades. Using Snell's re- 
fraction low and the continuous Dijkstra algorithm, Mitchell and Papadimitriou [19] present an $O\left(n^{8} \log \frac{n N^{\prime} \rho}{\epsilon}\right)$ time algorithm, where $N^{\prime}$ is the largest integer coordinate of vertices and $\rho$ is the ratio of the maximum weight to the minimum weight. Aleksandrov et al. [2, 3] provide two logarithmic discretization schemes that place Steiner points along edges or bisectors of angles of regions in $R$, forming a geometric progression. The placement of the Steiner points depends on an input parameter $\epsilon>0$ and the geometry of the subdivision. The $(1+\epsilon)$-approximation algorithms in $[2,3]$ take $O\left(\frac{n}{\epsilon}\left(\frac{1}{\sqrt{\epsilon}}+\log n\right) \log \frac{1}{\epsilon}\right)$ and $O\left(\frac{n}{\sqrt{\epsilon}} \log \frac{n}{\epsilon} \log \frac{1}{\epsilon}\right)$ time, respectively. Sun and Reif [21] give an algorithm, called BUSHWHACK, which constructs a discrete graph $G$ by placing Steiner points along edges of the subdivision. By exploiting the geometric property of an optimal path, BUSHWHACK computes an approximate path more efficiently as it accesses only a subgraph of $G$. Combined with the logarithmic discretization scheme introduced in [2], BUSHWHACK takes $O\left(\frac{n}{\epsilon}\left(\log \frac{1}{\epsilon}+\log n\right) \log \frac{1}{\epsilon}\right)$ time. Very recently, Aleksandrov et al. [1] gave a query algorithm that can find an $\epsilon$-approximate shortest path between any two points in $O(\bar{q})$ time, where $\bar{q}$ is a query time parameter. The preprocessing time of this algorithm is $O\left(\frac{(g+1) n^{2}}{\epsilon^{2 / 3} \bar{q}} \log \frac{n}{\epsilon} \log ^{4} \frac{1}{\epsilon}\right)$, where $g$ is the genus of the discrete graph constructed by the discretization scheme. Cheng et al. [10] give an algorithm to approximate optimal paths in anisotropic regions, which is a generalized case of weighted regions. Their algorithm takes $O\left(\frac{\rho^{2} \log \rho}{\epsilon^{2}} n^{3} \log \left(\frac{\rho n}{\rho}\right)\right)$ time, where $\rho \geq 1$ is a constant such that the convex distance function of any region contains a concentric Euclidean disk with radius $1 / \rho$. In weighted regions, the time complexity of the algorithm is improved to $O\left(\frac{\rho \log \rho}{\epsilon} n^{3} \log \left(\frac{\rho n}{\epsilon}\right)\right)$ time, where $\rho$ is the ratio of the maximum weight to the minimum weight. Very recently, Cheng et al. [11] also provided a query version of this algorithm that gives an approximate optimal path from a fixed source (in an anisotropic subdivision) in $O\left(\log \frac{\rho n}{\epsilon}\right)$ time. The preprocessing time is $O\left(\frac{\rho^{2} n^{4}}{\epsilon^{2}}\left(\log \frac{\rho n}{\epsilon}\right)^{2}\right)$.

\subsection{Definitions and Preliminaries}

We denote by $P(s) Q(t)$ the leash (line segment, also called link, or shortest path, depending of context) from $P(s)$ to $Q(t)$, where $s, t \in[0,1]$. 
Alt and Godau [4] introduced the free space diagram to solve the decision version of the non-geodesic Fréchet distance problem (unweighted case): Given two polygonal curves $P$ and $Q$, and a positive constant $\Delta$, determine if $\delta_{F}(P, Q)<\Delta$. A free space diagram is a $[0,1] \times[0,1]$ parameter space such that each point $(s, t)$ in the parameter space corresponds to a leash with end points $P(s)$ and $Q(t)$. A free space cell $C \subseteq[0,1]^{2}$ is defined by two line segments, one from each curve. $C$ corresponds to the leashes with endpoints on the two segments. The free space in the parameter space is defined as $\{(s, t): S(P(s), Q(t))<\Delta\}$. That is, the free space represents all links shorter than $\Delta$. A monotone path is a path monotone along both coordinate axes. There is a one-to-one correspondence between all possible matchings of $P$ and $Q$ and all monotone paths from $(0,0)$ to $(1,1)$ in the free space diagram. Hence, $\delta_{F}(P, Q)<\Delta$ if and only if there exists a monotone path in the free space from the bottom left corner to the top right corner of the parameter space.

The discrete Fréchet distance, introduced by Either and Mannila [15], considers only the vertices of the (polygonal) curves. A coupling $L$ between $\delta(P)$ and $\delta(Q)$ is defined as

$$
\left(u_{a_{1}}, v_{b_{1}}\right),\left(u_{a_{2}}, v_{b_{2}}\right), \ldots,\left(u_{a_{m}}, v_{b_{m}}\right)
$$

such that $a_{1}=1, b_{1}=1, a_{m}=p, b_{m}=q$, and for $i=1,2, \ldots, m$, we have $a_{i+1}=a_{i}$ or $a_{i+1}=a_{i}+1$, and $b_{i+1}=b_{i}$ or $b_{i+1}=b_{i}+1$. In other words, $L$ is an order preserving pairing of vertices in $P$ and $Q$. Note that each vertex can appear in $L$ more than once. The discrete Fréchet distance is defined as

$$
\delta_{d F}(P, Q)=\inf _{L} \max _{i=1,2, \ldots, m} S\left(u_{a_{i}}, v_{b_{i}}\right) .
$$

\subsection{Our results}

Let $\epsilon>0$ be a positive constant given as part of the input. We also assume $\epsilon<1$ at times. We have the following results: (1) For weighted regions in the plane, with $S(P(s), Q(t))=\sum_{i=1}^{n^{\prime}} w_{i} *$ $R_{i}(P(s) Q(t))$ (case (a) above), we present a $(1+\epsilon)$-approximation algorithm that takes $O\left(p q N^{4} \log (p q N)\right)$ time, $N=O\left(C(R)\left(\frac{n}{\epsilon}\left(\log \frac{1}{\epsilon}+\right.\right.\right.$ $\left.\log n) \log \frac{1}{\epsilon}\right)$ is the total number of Steiner points used and $C(R)$ is a constant associated with the geometry of the subdivision $R ;(2)$ 
For weighted regions in the plane, with $S(P(s), Q(t))=$ the length of the shortest (weighted) path from $P(s)$ to $Q(t)$, we present a $(1+\epsilon)$-approximation algorithm that takes $O\left(C(R)^{2} \frac{p q}{\epsilon^{2}}\left(\log ^{4} \frac{1}{\epsilon}\right) \bar{q}+\right.$ $\left.\frac{(g+1) n^{2}}{\epsilon^{2 / 3} \bar{q}} \log \frac{n}{\epsilon} \log ^{4} \frac{1}{\epsilon}\right)$ time, where $\bar{q}$ is a query time parameter related to computing shortest path and $g$ is the genus of the graph constructed by the discretization scheme. (3) We give a $(1+\epsilon)$-approximation algorithm for finding the Fréchet distance between $P$ and $Q$ among polyhedral obstacles in $\mathcal{R}^{3}$ ( 1 and $\infty$ weights). To the best of our knowledge this is the first result for the $\mathcal{R}^{3}$ problem. The algorithm takes $O\left(C(R)^{2} p q\left(1 / \epsilon^{2}\right) \log ^{4}(1 / \epsilon)\left(n^{2} \lambda(n) \log (n / \epsilon) / \epsilon^{4}+n^{2} \log (n \gamma) \log (n \log \gamma)\right)\right)$ time, where $\gamma$ is the ratio of the length of the longest obstacle edge to the Euclidean distance between the two points, and $\lambda(n)$ is a very slowly-growing function related to the inverse of the Ackermann's function.

\section{The Line Segment Leash}

In this section, we discuss the case of the line segment leash. That is, $S(P(s), Q(t))=\sum_{i=1}^{n^{\prime}} w_{i} * R_{i}(P(s) Q(t))$. We first briefly discuss a pseudo polynomial exact algorithm for solving the decision version of this problem. Then, we address the optimization version of this problem directly and give a polynomial time approximation algorithm.

\subsection{An Exact Algorithm for the Decision Problem}

In this section, we give an exact algorithm extending Alt and Godau's algorithm [4] to solve the decision version of the problem. Recall that the free space diagram introduced by Alt and Godau is a $[0,1] \times[0,1]$ parameter space such that each point $(s, t)$ in the parameter space corresponds to a leash with end points $P(s)$ and $Q(t)$. The free space diagram can be decomposed into $O(p q)$ free space cells such that each cell $C$ corresponds to the leashes with endpoints on two segments, one from each curve, where $p$ and $q$ are the number of vertices of $P$ and $Q$, respectively. To determine if $\delta_{F}(P, Q)<\Delta$ for some positive constant $\Delta$, we proceed as follows: 


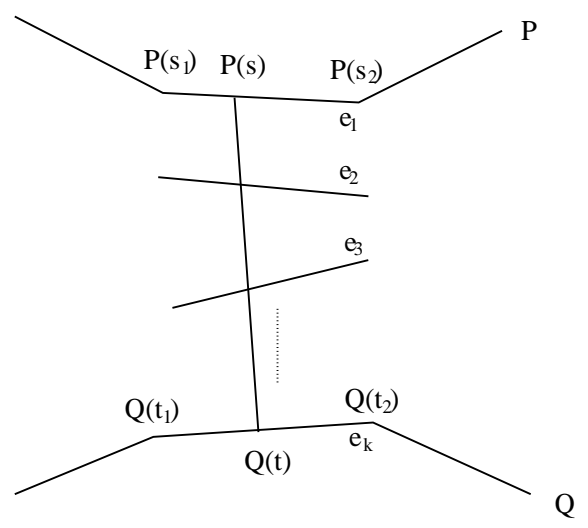

Fig. 2. A leash intersects with a sequence of edges.

1. Partition each cell $C$ into regions such that each region corresponds to leashes intersecting the same sequence of edges in $R$.

2. For each region, find the free space with respect to the positive constant $\Delta$ by solving an $O(n)$ order bivariate polynomial system with $O(n)$ equations and inequalities.

3. Determine if there exists a monotone path in the free space from $(0,0)$ to $(1,1)$.

The partition in step 1 is an arrangement of $O(n)$ curves of the form $s t+c_{1} s+c_{2} t+c_{3}=0$, where $c_{1}, c_{2}$, and $c_{3}$ are constants. In the worst case, the total number of regions in the parameter space is $\left(p q n^{2}\right)$. Each region can be defined mathematically by $O(n)$ inequalities. Once the free space in each region is computed, step 3 can be solved by decomposing the parameter space and constructing a directed shortest path graph. We will address steps 1 and 3 in detail in later sections. Both steps can be solved in polynomial time. Next, we will show that Step 2 can be solved, which dominates the complexity of this algorithm.

For each region, let $f(s, t)$ be the weighted length of the leash $P(s) Q(t)$ in terms of $s$ and $t$, let and $\left\{e_{1}, e_{2}, \ldots, e_{k}\right\}$ be the sequence of edges intersected by the leash. Note that $k=O(n)$ and $e_{1}$ and $e_{k}$ are the two segments in $P$ and $Q$, respectively. Following [9], the weighted length of leashes within the same region have the same functional expression, i.e. $S(P(s), Q(t))=\sqrt{1+m^{2}} \sum_{i=1}^{k-1} w_{i} \mid \frac{p_{i+1}-p}{m-m_{i+1}}-$ $\frac{p_{i}-p}{m-m_{i}} \mid=\sqrt{1+m^{2}} \sum_{i=1}^{k} \frac{a_{i} p+b_{i}}{m-m_{i}}$, where $a_{i}, b_{i}$ are constants, $w_{i}$ is the 
weight of the region bounded by edges $e_{i}$ and $e_{i+1}, m, p$ are the slope and intercept of the leash, respectively, and $m_{i}, p_{i}$ are the slope and intercept of $e_{i} \in\left\{e_{1}, e_{2}, \ldots, e_{k}\right\}$, respectively. Let the end points of $e_{1}$ be $P\left(s_{1}\right)=\left(x_{1}, y_{1}\right)$ and $P\left(s_{2}\right)=\left(x_{2}, y_{2}\right)$, respectively, and the end points of $e_{k}$ be $Q\left(t_{1}\right)=\left(x_{3}, y_{3}\right)$ and $Q\left(t_{2}\right)=\left(x_{4}, y_{4}\right)$, respectively. For any $s \in\left[s_{1}, s_{2}\right]$, we have

$P(s)=\left(x_{1}+\left(x_{2}-x_{1}\right)\left(s-s_{1}\right) /\left(s_{2}-s_{1}\right), y_{1}+\left(y_{2}-y_{1}\right)\left(s-s_{1}\right) /\left(s_{2}-s_{1}\right)\right)$, and similarly for any $t \in\left[t_{1}, t_{2}\right]$, we have

$Q(t)=\left(x_{3}+\left(x_{4}-x_{3}\right)\left(t-t_{1}\right) /\left(t_{2}-t_{1}\right), y_{3}+\left(y_{4}-y_{3}\right)\left(t-t_{1}\right) /\left(t_{2}-t_{1}\right)\right)$.

Expressing $m$ and $p$ in terms of $s$ and $t$, we obtain that

$$
m=\frac{c_{1} s+c_{2} t+c_{3}}{d_{1} s+d_{2} t+d_{3}}
$$

and

$$
p=\frac{c_{1}^{\prime} s t+c_{2}^{\prime} s+c_{3}^{\prime} t+c_{4}^{\prime}}{d_{1} s+d_{2} t+d_{3}}
$$

where $c_{1}, c_{2}, c_{3}, c_{1}^{\prime}, c_{2}^{\prime}, c_{3}^{\prime}, c_{4}^{\prime}, d_{1}, d_{2}$, and $d_{3}$ are constants. It follows that

$f(s, t)=\sqrt{1+\left(\frac{c_{1} s+c_{2} t+c_{3}}{d_{1} s+d_{2} t+d_{3}}\right)^{2}} \sum_{i=1}^{k} \frac{a_{i}\left(c_{1}^{\prime} s t+c_{2}^{\prime} s+c_{3}^{\prime} t+c_{4}^{\prime}\right) /\left(d_{1} s+d_{2} t+d_{3}\right)+b_{i}}{\left(c_{1} s+c_{2} t+c_{3}\right) /\left(d_{1} s+d_{2} t+d_{3}\right)-m_{i}}$.

We can find the boundary of free space in this region by solving the following equation system: $f(s, t)=\Delta$ subjects to $O(n)$ inequalities that define the region.

Since there are $O(n)$ fractional terms in $f(s, t)$, we can convert this equation system into a bivariate polynomial system of degree $O(n)$ which consists of one polynomial equation and $O(n)$ inequalities and requires pseudo polynomial time to solve [13].

We use Collins' cylindrical algebraic decomposition technique [13] analyzing the polynomial system, which takes $O\left((\bar{m} \bar{n})^{k^{r}} d^{k}\right)$ time, where $\bar{m}$ is the number of equation and inequalities in the polynomial system, $\bar{n}$ is the maximum degree of polynomials, $r$ is the number of variables, $k$ is a constant and $d$ is the maximum bit length of coefficients. Hence, step 2 takes $O\left(p q n^{2} n^{O(1)} d^{O(1)}\right)$ time.

This exact algorithm is not the one which ensures practical application. Next, we are going propose an approximation algorithm 
for the optimization version of this problem. To approximate the Fréchet distance, we discretize continuous space by placing Steiner points on edges of $R$. We place the Steiner points in such a way that all links can be grouped into sets. In each set, the difference between weighted lengths of any two links is small. Instead of finding the optimal matching, we find the sequence of sets that the leash traversed in a specific matching. We then pick an arbitrary link from each set in the sequence, the maximum link length gives an approximation of the Fréchet distance.

\subsection{Discretization Using Steiner Points}

We first discretize the continuous space by placing Steiner points in $R$ extending the discretization scheme given in [2]. Let $E$ be the set of all edges in $R$. Let $V$ be the set of vertices in $R$. For any point $v$ on an edge in $E$, let $E(v)$ be the set of edges incident to $v$ and let $d(v)$ be the minimum distance between $v$ and edges in $E \backslash E(v)$. For each edge $e \in E$, let $d(e)=\sup \{d(v) \mid v \in e\}$ and let $v_{e}$ be the point on $e$ so that $d\left(v_{e}\right)=d(e)$. For each $v \in V$, the vertex radius for $v$ is defined as $r(v)=\frac{\epsilon B}{n w_{\max }(v)}$, where $\epsilon$ is a positive real number defining the quality of the approximation, $B$ is a lower bound on $\delta_{F}(P, Q)$, and $w_{\max }(v)$ is the maximum weight among all weighted regions incident to $v$. The disk of radius $r(v)$ centered at $v$ defines the vertex-vicinity of $v . B$ can be computed by setting weights of all regions to the minimum weight of $R$ (assume all weights are greater than zero) and applying the continuous Fréchet distance algorithm described in [4], where $p$ and $q$ are the number of vertices of the curves $P$ and $Q$, respectively.

For each edge $e=v_{1} v_{2}$ in $E$, we place Steiner points $v_{i, 1}, v_{i, 2}, \ldots, v_{i, k_{i}}$ outside the vertex-vicinity of $v_{i}$, for $i=1,2$, such that $\left|v_{i} v_{i, 1}\right|=r\left(v_{i}\right)$, $\left|v_{i, j} v_{i, j+1}\right|=\epsilon d\left(v_{i, j}\right)$, for $j=1,2, \ldots, k_{i}-1$, and $v_{i, k_{i}}=v_{e}$. It follows from [2] that the number of Steiner points placed on an edge is $O(C(e) 1 / \epsilon \log 1 / \epsilon)$, where $C(e)=O\left(\frac{|e|}{d(e)} \log \frac{|e|}{\sqrt{r\left(v_{1}\right) r\left(v_{2}\right)}}\right)$. Let $N$ denote the total number of Steiner points and vertices of $R$. Since we set $r(v)=\frac{\epsilon B}{n w_{\max }(v)}$ for each $v \in R$, we have $N=O\left(C(R)\left(\frac{n}{\epsilon}\left(\log \frac{1}{\epsilon}+\right.\right.\right.$ $\left.\left.\log n) \log \frac{1}{\epsilon}\right)\right)$, where $C(R)=\max _{e \in R}\left(\frac{|e| \log |e| w_{\max }(e)}{d(e) B}\right)$ is a function as- 


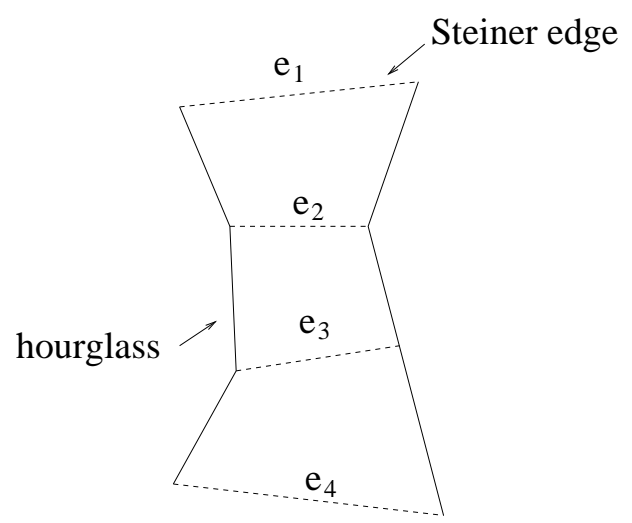

Fig. 3. An hourglass is the union of leashes intersecting with the same set of Steiner edges.

sociated with the geometry of $R$, and $w_{\max }(e)$ is the maximum weight among all weighted regions incident to the end points of $e$.

We refer to a line segment bounded by two consecutive Steiner points on an edge of $R$ as a Steiner edge. An hourglass is the union of all leashes (line segments) intersecting the same sequence of Steiner edges in the same order. Let $H$ be an hourglass defined by a sequence of Steiner edges $\left\{e_{1}, e_{2}, \ldots, e_{k}\right\}$, where $e_{1} \in P$ and $e_{k} \in Q$ (See Fig. 3).

Lemma 1. Let $l$ and $l^{\prime}$ be two segments in $H$. Then, $S(l) \leq(1+$ $2 \epsilon) S\left(l^{\prime}\right)+2 \epsilon B$.

Proof. Let $R_{i_{1}}, R_{i_{2}}, \ldots, R_{i_{k-1}}$ be the weighted regions in $H$, such that $R_{i_{j}}$ is between $e_{j}$ and $e_{j+1}$. Recall that for a region $R_{k}$ of $R, R_{k}(l)$ denotes the Euclidean length of $l$ within $R_{k}$. We have

$$
S(l)=\sum_{j=1}^{k-1} w_{i_{j}} R_{i_{j}}(l) \leq \sum_{j=1}^{k-1} w_{i_{j}}\left(R_{i_{j}}\left(l^{\prime}\right)+e_{j}+e_{j+1}\right),
$$

where $w_{i_{j}}$ is the positive weight associated with $R_{i_{j}}$.

If a Steiner edge $e_{j}$ is outside of any vertex-vicinity, $\left|e_{j}\right| \leq \epsilon R_{i_{j-1}}\left(l^{\prime}\right)$ and $\left|e_{j}\right| \leq \epsilon R_{i_{j}}\left(l^{\prime}\right)$, due to the placement of the Steiner points. If $e_{j}$ is incident to a vertex $v$, then $\left|e_{j}\right|=r(v)=(\epsilon B) /\left(n w_{\max }(v)\right)$. One segment can intersect at most $O(n)$ Steiner edges inside vertex vicinities. The result follows. 
Let $l_{H}$ be an arbitrary segment in $H$, with endpoints on $P$ and $Q$. Let $\alpha$ and $\beta$ be two reparametrizations that define a matching between $P$ and $Q$. Let $J=\left\{H_{1}, H_{2}, \ldots, H_{k}\right\}$ be the set of hourglasses traversed by the leash $P(\alpha(r)) Q(\beta(r))$. For an hourglass $H \in J$, let $I_{H}=\{e \mid e=P(\alpha(r)) Q(\beta(r)), r \in[0,1], e \in H\}$. Let $H(\alpha, \beta)$ be the segment in $I_{H}$ with the largest weighted length. That is, $S(H(\alpha, \beta))=\max _{e \in I_{H}} S(e)$. Let $\delta(\alpha, \beta)=\sup _{r \in[0,1]} S(P(\alpha(r)), Q(\beta(r)))$.

Lemma 2. $\left|\max _{H \in J} S\left(l_{H}\right)-\delta(\alpha, \beta)\right| \leq 4 \epsilon \delta(\alpha, \beta)$.

Proof. Applying Lemma 1, we have,

$$
S\left(l_{H}\right) \leq(1+2 \epsilon) S(H(\alpha, \beta))+2 \epsilon D,
$$

and

$$
S(H(\alpha, \beta)) \leq(1+2 \epsilon) S\left(l_{H}\right)+2 \epsilon D .
$$

Assume $\epsilon<1 / 2$, it follows,

$$
\max _{H \in J} S\left(l_{H}\right) \geq \frac{1-2 \epsilon}{1+2 \epsilon} \delta(\alpha, \beta) \geq(1-4 \epsilon) \delta(\alpha, \beta),
$$

and

$$
\max _{H \in J} S\left(l_{H}\right) \leq(1+2 \epsilon) \max _{H \in J} S(H(\alpha, \beta))+2 \epsilon D \leq(1+4 \epsilon) \delta(\alpha, \beta) .
$$

We say that $\delta(J)=\max _{H \in J} S\left(l_{H}\right)$ is a $4 \epsilon$-approximation of $\delta(\alpha, \beta)$. Given a sequence of hourglasses $J=\left\{H_{1}, H_{2}, \ldots, H_{k}\right\}$, we call $J$ a legal sequence if there exists two reparmetrizations $\alpha, \beta$ that define a leash traversing the same sequence of hourglasses as $J$.

Lemma 3. We can find a value $\delta_{F}^{\prime}(P, Q)$ such that $\mid \delta_{F}^{\prime}(P, Q)$ $\delta_{F}(P, Q) \mid \leq 4 \epsilon \delta_{F}(P, Q)$, that is, $\delta_{F}^{\prime}(P, Q)$ is a $4 \epsilon$-approximation of $\delta_{F}(P, Q)$.

Proof. Let $\hat{\alpha}, \hat{\beta}$ be the optimal reparametrizations that give the Fréchet distance between $P$ and $Q$. Let $\hat{J}$ be the sequence of hourglasses traversed by the leash defined by $\alpha$ and $\beta$. Applying Lemma 2, we have,

$$
|\delta(\hat{J})-\delta(\hat{\alpha}, \hat{\beta})|=\left|\delta(\hat{J})-\delta_{F}(P, Q)\right| \leq 4 \epsilon \delta(\hat{\alpha}, \hat{\beta})=4 \epsilon \delta_{F}(P, Q) .
$$

So, there is a legal sequence of hourglasses that gives a $4 \epsilon$-approximation of $\delta_{F}(P, Q)$. 


\subsection{Fréchet Distance Between Two Segments}

Next, for simplicity, we study a special case of the problem, in which each curve consists of one line segment. See Fig. 4 for an illustration. Similar to Alt and Godau's algorithm, we attack the problem in the parameter space $D=[0,1]^{2}$, where a leash $P(s) Q(t)$ is associated with a point $(s, t) \in D$. We refer to $(s, t) \in D$ as the dual point of the leash $P(s) Q(t)$.

Lemma 4. All leashes through a Steiner point $v$ correspond to a curve in $D$, with equation $C_{v}: s t+c_{1} s+c_{2} t+c_{3}=0$, where $c_{1}, c_{2}$, and $c_{3}$ are constants.

Proof. Let $v=(a, b), P(0)=\left(x_{1}, y_{1}\right), P(1)=\left(x_{2}, y_{2}\right), Q(0)=$ $\left(x_{3}, y_{3}\right), Q(1)=\left(x_{4}, y_{4}\right)$. We have

$$
\begin{aligned}
& P(s)=\left(x_{1}+\left(x_{2}-x_{1}\right) s, y_{1}+\left(y_{2}-y_{1}\right) s\right), \\
& Q(t)=\left(x_{3}+\left(x_{4}-x_{3}\right) t, y_{3}+\left(y_{4}-y_{3}\right) t\right),
\end{aligned}
$$

and

$P(s) Q(t): y-\left(y_{1}+\left(y_{2}-y_{1}\right) s\right)=\frac{y_{1}+\left(y_{2}-y_{1}\right) s-\left(y_{3}+\left(y_{4}-y_{3}\right) t\right)}{x_{1}+\left(x_{2}-x_{1}\right) s-\left(x_{3}+\left(x_{4}-x_{3}\right) t\right)}\left(x-\left(x_{1}+\right.\right.$ $\left.\left.\left(x_{2}-x_{1}\right) s\right)\right)$.

Since $P(s) Q(t)$ passes through $v$, we obtain that

$\left.\left(b-y_{1}-\left(y_{2}-y_{1}\right) s\right)\left(x_{1}-x_{3}+\left(x_{2}-x_{1}\right) s-\left(x_{4}-x_{3}\right) t\right)\right)=\left(a-x_{1}-\right.$ $\left.\left(x_{2}-x_{1}\right) s\right)\left(y_{1}-y_{3}+\left(y_{2}-y_{1}\right) s-\left(y_{4}-y_{3}\right) t\right)$,

and thus we have

$$
C_{v}: s t+c_{1} s+c_{2} t+c_{3}=0,
$$

where $c_{1}, c_{2}$, and $c_{3}$ are constants.

We call the curve $C_{v}$ the dual curve of Steiner point $v \cdot C_{v}$ is continuous and monotone along both (s and t) axes. Obviously, $v$ lies on a leash if and only if the dual point of the leash in $D$ lies on $C_{v}$. Next we define the relative position of two leashes with respect to a Steiner point $v$. Given two leashes $P(s) Q(t)$ and $P\left(s^{\prime}\right) Q\left(t^{\prime}\right)$, we say they are on the same side of $v$ if and only if there exists two continuous functions $\alpha^{\prime}, \beta^{\prime}:[0,1] \rightarrow[0,1]$, such that $\alpha^{\prime}(0)=s$, $\alpha^{\prime}(1)=s^{\prime}, \beta^{\prime}(0)=t, \beta^{\prime}(1)=t^{\prime}$ and $v \notin P\left(\alpha^{\prime}(r) Q\left(\beta^{\prime}(r)\right), \forall r \in[0,1]\right.$. Note that $\alpha^{\prime}, \beta^{\prime}$ are not necessarily monotone. Intuitively, two leashes 


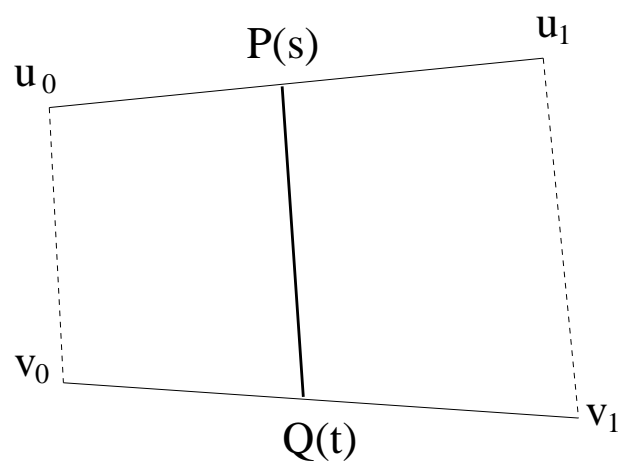

Fig. 4. A special case of the Fréchet distance problem.

are on the same side of $v$, if one leash can transform to the other one by sweeping its end points on their respective curves without crossing $v$.

Lemma 5. $C_{v}$ divides $D$ into two partitions, each partition corresponding to all links on the same side of point $v$.

Proof. Given two links $P(s) Q(t)$ and $P\left(s^{\prime}\right) Q\left(t^{\prime}\right)$, since there is a oneto-one correspondence between all possible walks from $P(s) Q(t)$ to $P\left(s^{\prime}\right) Q\left(t^{\prime}\right)$ and all paths from $(s, t)$ to $\left(s^{\prime}, t^{\prime}\right)$ in $D$, if $(s, t),\left(s^{\prime}, t^{\prime}\right)$ are on different sides of $C_{v}$, all paths between $(s, t)$ and $\left(s^{\prime}, t^{\prime}\right)$ must pass $C_{v}$ at least once. That is, no walk exists between the two links without crossing $v$.

We partition $D$ by the dual curves of the Steiner points. Using the algorithm in [5], the partition can be computed in $O(N \log N+k)$ time and $O(N+k)$ space, where $N=O\left(C(R)\left(\frac{n}{\epsilon}\left(\log \frac{1}{\epsilon}+\log n\right) \log \frac{1}{\epsilon}\right)\right)$ is the total number of Steiner points and $k$ is the number of cells, which is $O\left(N^{2}\right)$ in the worst case. Let $A$ denote the partition.

Lemma 6. In A, each cell corresponds to an hourglass.

Proof. If two points $(s, t),\left(s^{\prime}, t^{\prime}\right)$ belong to the same cell, then there exists a walk between $P(s) Q(t)$ and $P\left(s^{\prime}\right) Q\left(t^{\prime}\right)$ that does not cross any Steiner point, i.e. $P(s) Q(t)$ and $P\left(s^{\prime}\right) Q\left(t^{\prime}\right)$ intersect with the same set of Steiner edges. If $(s, t),\left(s^{\prime}, t^{\prime}\right)$ belong to different cells, then any walk between $P(s) Q(t)$ and $P\left(s^{\prime}\right) Q\left(t^{\prime}\right)$ must cross at least 
one Steiner point. That is, $P(s) Q(t), P\left(s^{\prime}\right) Q\left(t^{\prime}\right)$ do not intersect with the same set of Steiner edges. The result follows.

The Fréchet distance between $P$ and $Q$ can then be approximated as follows:

1. Place Steiner points as described previously.

2. Partition $D$ by dual curves of all Steiner points.

3. For each cell $Z$, choose an arbitrary leash $l$ and assign $S(l)$ as the weight of the cell.

4. Find a monotone path $T$ in $D$, from its left bottom corner, $(0,0)$, to its top right corner, $(1,1)$, such that the maximum weight of the cells traversed by $T$ is minimized.

Since the sequence of cells traversed by a monotone path in $D$ corresponds to a legal sequence of hourglasses traversed by a leash following a match of $P$ and $Q$, and vice versa, by Lemma 3 the maximum weight of the cells traversed by $T$ is a $4 \epsilon$-approximation of the Fréchet distance between $P$ and $Q$.

\subsection{Finding an optimal path in $D$}

We define the cost of a path between two points in $D$ as the maximum weight of the regions traversed by the path. We decompose $D$ by extending a horizontal as well as a vertical line from every vertex until it reaches the boundary of $D$. See Fig. 6 for an illustration. Let the new subdivision be $D^{\prime}$.

Lemma 7. Given an edge e in $D^{\prime}$, let $p$ be a point on e and let $T_{p}$ be an arbitrary monotone path from $(0,0)$, i.e. the bottom left corner of $D^{\prime}$, to $p$. Then, there exists a monotone path from $(0,0)$ to any point on $e$ with the same cost of $T_{p}$.

Proof. We consider $e$ in $D$. Note that $D$ consists of dual lines of Steiner points only. In $D$, we extend a vertical line as well as a horizontal line from the end points of $e$. Let $L_{1}, L_{2}$ be the two horizontal lines which define a horizontal slab and $L_{3}, L_{4}$ be the two vertical lines which define a vertical slab. Obviously, $T_{p}$ has the same cost in $D$ as it does in $D^{\prime}$. Without loss of generality, assume $T_{v}$ enters the horizontal slab before it enters the vertical slab. Let $v$ be the entry 


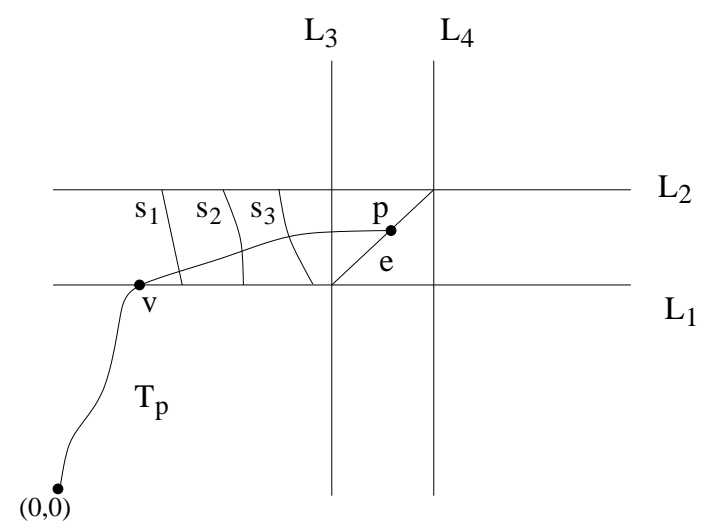

Fig. 5. There exists a monotone path from $(0,0)$ to every point in $e$, which has the same cost as $T_{p}$.

point. Let $S=\left\{s_{1}, s_{2}, \ldots, s_{k}\right\}$ be the set of curves on edges of $D$, which are bounded by the horizontal slab and traversed by $T_{p}$. Since the horizontal slab does not contain any vertex of $D$ in its interior, no two curves in $S$ intersect with each other. Hence we can construct a monotone path from $v$ to any point on $e$ that traverses $S$, i.e. we can contract a monotone path from $(0,0)$ to any point on $e$ such that it has the same cost as $T_{p}$. See Fig. 5 for an illustration. The same argument holds if $T_{p}$ enters the vertical slab first.

Thus, given an optimal monotone path $T_{p}$ from $(0,0)$ to an arbitrary point $p \in e$, we can construct a monotone path from $(0,0)$ to any point in $e$, which has the same cost as $T_{p}$ and is also optimal. To find the optimal monotone path from $(0,0)$ to $(1,1)$, we construct a directed graph $G$ having edges of $D^{\prime}$ as vertices. In $G$, a directed edge is added from a node $v_{1}$ to a node $v_{2}$ if, in $D^{\prime}, v_{1}$ and $v_{2}$ are on the boundary of the same cell and there exists a monotone path from $v_{1}$ to $v_{2}$. The optimal monotone path can be found by running a modified Dijkstra's algorithm on $G$.

Time complexity: The complexity of $D^{\prime}$ is $N^{4}$, where $N=O\left(C(R)\left(\frac{n}{\epsilon}\left(\log \frac{1}{\epsilon}+\right.\right.\right.$ $\left.\left.\log n) \log \frac{1}{\epsilon}\right)\right)$ is the number of Steiner points used, since each cell in $D^{\prime}$ has $O(1)$ edges and vertices, and thus each cell contributes $O(1)$ 
edges to $G$. Then, $G$ has $O\left(N^{4}\right)$ vertices and $O\left(N^{4}\right)$ edges and Dijkstra's algorithm takes $O\left(N^{4} \log N\right)$ time.

\subsection{Fréchet Distance Between Two Polygonal curves}

We extend the algorithm above to approximate the Fréchet distance between two polygonal chains $P$ and $Q$. Recall that $p$ and $q$ are the number of vertices of $P$ and $Q$, respectively. The parameter space $D$ can be divided into $(p-1)(q-1)$ subspaces, such that each subspace corresponds to all leashes bounded by the same two segments, one from each chain. Each subspace can be partitioned independently by introducing dual curves of Steiner points. The Fréchet distance $\delta_{F}(P, Q)$ can be approximated by finding an optimal monotone path from the bottom left corner to the top right corner of $D$. The complexity of $D$ is $O\left(p q N^{2}\right)$ and the complexity of the decomposed parameter space $D^{\prime}$ is $O\left(p^{2} q^{2} N^{4}\right)$, where

$N=O\left(C(R)\left(\frac{n}{\epsilon}\left(\log \frac{1}{\epsilon}+\log n\right) \log \frac{1}{\epsilon}\right)\right)$ is the total number of Steiner points. It takes $O\left(p^{2} q^{2} N^{4} \log (p q N)\right)$ time to approximate $\delta_{F}(P, Q)$.

Theorem 1. An approximation of the Fréchet distance between two polygonal curves in a weighted subdivision can be computed in $O\left(p^{2} q^{2} N^{4} \log (p q N)\right)$ time, where $N=O\left(C(R)\left(\frac{n}{\epsilon}\left(\log \frac{1}{\epsilon}+\log n\right) \log \frac{1}{\epsilon}\right)\right)$ is the total number of Steiner points.

\section{Geodesic Fréchet Distance}

In this section we study two versions of the geodesic Fréchet distance problem. We do not require the leash to be homotopic, i.e. the leash is allowed to sweep discontinuously without penalty. For example, the leash can pass through or jump over obstacles.

\subsection{Geodesic Fréchet Distance in Weighted Regions in $\mathcal{R}^{2}$}

Recall that the cost (weighted length) of a path in $R$ is defined as the weighted sum of its Euclidean lengths within each region of $R$. A geodesic path between two points in $R$ is a path between those points that has minimum cost. Here, the distance between two points in $R$ is the cost of the geodesic path between those points. 


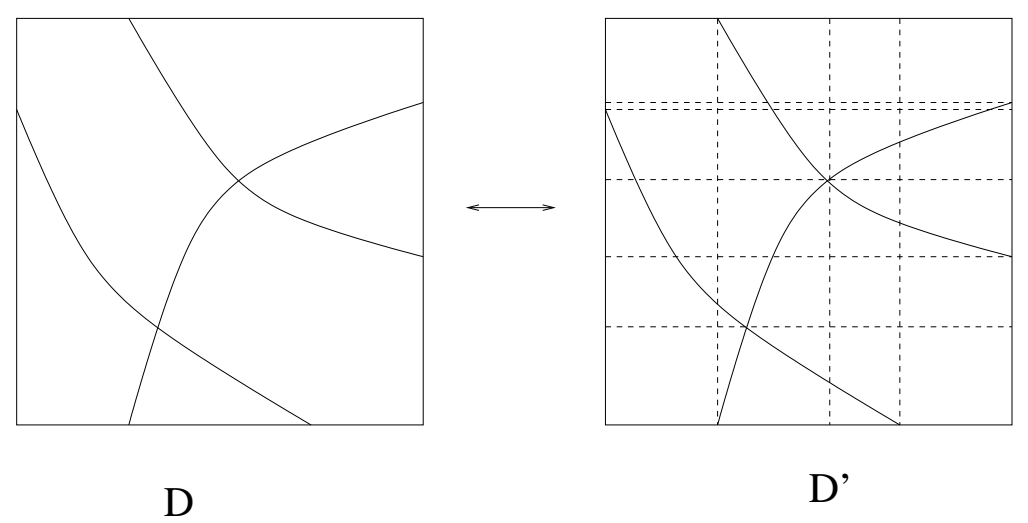

Fig. 6. Decomposition of $D$.

We prove that the geodesic Fréchet distance can be approximated by the discrete geodesic Fréchet distance. Given two polygonal curves $P, Q$, to approximate $\delta_{F}(P, Q)$, we need to add additional vertices to $P$ and $Q$. We follow a similar approach as in [2], except that we define the vertex radius of a vertex $v$ in $P$ or $Q$ as $r(v)=\frac{\epsilon B}{w_{\max }(v)}$, where $\epsilon$ is a positive real number defining the quality of the approximation, $B$ is a lower bound on $\delta_{F}(P, Q)$, and $w_{\max }(v)$ is the maximum weight of regions incident to $v$. Recall that the disk of radius $r(v)$ centered at $v$ defines the vertex-vicinity of $v$. New vertices are placed on edges of $P$ nd $Q$ forming a geometric progression with ratios depending on $\epsilon$ and on the geometry of $R$ (see Section 3.1 for details). The total number of additional vertices introduced on each edge is $O\left(C(R) \frac{1}{\epsilon} \log ^{2} \frac{1}{\epsilon}\right)$, where $C(R)$ is a constant associated with geometry of $R$. Let $P^{\prime}$ and $Q^{\prime}$ be the new polygonal chains and let $p^{\prime}=O\left(C(R) \frac{p}{\epsilon} \log ^{2} \frac{1}{\epsilon}\right)$ and $q^{\prime}=O\left(C(R) \frac{q}{\epsilon} \log ^{2} \frac{1}{\epsilon}\right)$ be the number of vertices of $P^{\prime}$ and $Q^{\prime}$, respectively.

Lemma 8. The discrete geodesic Fréchet distance between $P^{\prime}$ and $Q^{\prime}$ gives an $\epsilon$-approximation of the geodesic Fréchet distance between $P$ and $Q$, i.e. $(1-\epsilon) \delta_{F}(P, Q) \leq \delta_{d F}\left(P^{\prime}, Q^{\prime}\right) \leq(1+\epsilon) \delta_{F}(P, Q)$.

Proof. Here, $d(a, b)$ denotes the weighted length of the line segment $a b$. Let $\alpha, \beta$ be two optimal reparametrizations between $P^{\prime}$ and $Q^{\prime}$. Obviously, they are also an optimal matching between $P$ and $Q$. Following [15], we define a coupling $L$ between $\delta\left(P^{\prime}\right)=\left\{u_{1}, u_{2}, \ldots, u_{p^{\prime}}\right\}$ 
and $\delta\left(Q^{\prime}\right)=\left\{v_{1}, v_{2}, \ldots, v_{q^{\prime}}\right\}$. For each point $u \in \delta\left(P^{\prime}\right)$, let $t(u)$ be the smallest value such that $P^{\prime}(\alpha(t(u)))=u$, and for each point $v$ in $\delta\left(Q^{\prime}\right)$, let $s(v)$ be the smallest value such that $Q^{\prime}(\beta(s(v)))=v$. First, we add $\left(u_{1}, v_{1}\right)$ to $L$. Let $i$ and $j$ be the number of distinct points in $\delta\left(P^{\prime}\right)$ and $\delta\left(Q^{\prime}\right)$, respectively, added to $R$. If $j=q^{\prime}$ or $t\left(u_{i+1}\right) \leq s\left(v_{j+1}\right)$, then add $\left(u_{i+1}, v_{j}\right)$ to $L$. We have

$$
d\left(u_{i+1}, v_{j}\right) \leq S\left(P^{\prime}\left(\alpha\left(t\left(u_{i+1}\right)\right)\right) Q^{\prime}\left(\beta\left(t\left(u_{i+1}\right)\right)\right)\right)+d\left(v_{j}, v_{j+1}\right)
$$

and

$$
S\left(P^{\prime}\left(\alpha\left(t\left(u_{i+1}\right)\right)\right) Q^{\prime}\left(\beta\left(t\left(u_{i+1}\right)\right)\right)\right) \leq d\left(u_{i+1}, v_{j}\right)+d\left(v_{j}, v_{j+1}\right) .
$$

If $v_{j} v_{j+1}$ is not contained in any vertex vicinity, we have

$$
d\left(v_{j}, v_{j+1}\right) \leq \epsilon S\left(P^{\prime}\left(\alpha\left(t\left(u_{i+1}\right)\right)\right), Q^{\prime}\left(\beta\left(t\left(u_{i+1}\right)\right)\right)\right) \leq \epsilon \delta_{F}(P, Q) .
$$

If $v_{j} v_{j+1}$ is inside a vertex vicinity, we have

$$
d\left(v_{j}, v_{j+1}\right) \leq \epsilon B \leq \epsilon \delta_{F}(P, Q) .
$$

We obtain that

$$
\left|S\left(P^{\prime}\left(\alpha\left(t\left(u_{i+1}\right)\right)\right), Q^{\prime}\left(\beta\left(t\left(u_{i+1}\right)\right)\right)\right)-d\left(u_{i+1}, v_{j}\right)\right| \leq \epsilon \delta_{F}(P, Q) .
$$

If $i=p^{\prime}$ or $t\left(u_{i+1}\right)>s\left(v_{j+1}\right)$, then we add $\left(u_{i}, v_{j+1}\right)$ to $L$. Similarly, we have

$$
\left|S\left(P^{\prime}\left(\alpha\left(s\left(v_{j+1}\right)\right)\right), Q^{\prime}\left(\beta\left(s\left(v_{j+1}\right)\right)\right)\right)-d\left(u_{i}, v_{j+1}\right)\right| \leq \epsilon \delta_{F}(P, Q),
$$

and thus

$$
(1-\epsilon) \delta_{F}(P, Q) \leq \delta_{d F}\left(P^{\prime}, Q^{\prime}\right) \leq(1+\epsilon) \delta_{F}(P, Q)
$$

Instead of finding an exact geodesic path between two points, we can apply existing shortest path algorithms in weighted regions, which find an approximate path. Let $\delta_{d F}^{\prime}\left(P^{\prime}, Q^{\prime}\right)$ be the approximate discrete Fréchet distance computed by replacing the exact shortest 
path algorithm by the approximation algorithm, which gives an $\epsilon$ approximation of the geodesic distance between points. We have

$\delta_{d F}^{\prime}\left(P^{\prime}, Q^{\prime}\right) \leq(1+\epsilon) \delta_{d} F(P, Q) \leq(1+\epsilon)^{2} \delta_{F}(P, Q) \leq(1+3 \epsilon) \delta_{F}(P, Q)$.

Similarly, assuming that $\epsilon \leq 1 / 3$, we have

$$
\delta_{d F}^{\prime}\left(P^{\prime}, Q^{\prime}\right) \geq(1-3 \epsilon) \delta_{F}(P, Q) .
$$

Thus, we obtain

$$
\left|\delta_{d F}^{\prime}\left(P^{\prime}, Q^{\prime}\right)-\delta_{F}(P, Q)\right| \leq 3 \epsilon \delta_{F}(P, Q) .
$$

If a non-query based approximation algorithm is used, $\delta_{d F}^{\prime}\left(P^{\prime}, Q^{\prime}\right)$ can be computed in time $O\left(p^{\prime} q^{\prime} T(n, \epsilon)\right)=O\left(C(R)^{2} \frac{p q}{\epsilon^{2}}\left(\log ^{4} \frac{1}{\epsilon}\right) T(n, \epsilon)\right)$, where $T(n, \epsilon)$ is the time to compute an approximate shortest path between two points. If a query-based approximation algorithm is used, then $\delta_{d F}^{\prime}\left(P^{\prime}, Q^{\prime}\right)$ can be computed in $O\left(p^{\prime} q^{\prime} Q U E R Y(n, \epsilon)+P R E(n, \epsilon)\right)=O\left(C(R)^{2} \frac{p q}{\epsilon^{2}}\left(\log ^{4} \frac{1}{\epsilon}\right) Q U E R Y(n, \epsilon)+\right.$ $\operatorname{PRE}(n, \epsilon))$, where $Q U E R Y(n, \epsilon)$ is the query time and $\operatorname{PRE}(n, \epsilon)$ is the preprocessing time of the algorithm. For example, if we use the algorithm proposed by Aleksandrov et. al. [1], the algorithm takes $O\left(C(R)^{2} \frac{p q}{\epsilon^{2}}\left(\log ^{4} \frac{1}{\epsilon}\right) \bar{q}+\frac{(g+1) n^{2}}{\epsilon^{2 / 3} \bar{q}} \log \frac{n}{\epsilon} \log ^{4} \frac{1}{\epsilon}\right)$ time, where $\bar{q}$ is a query time parameter and $g$ is the genus of the graph constructed by the discretization scheme.

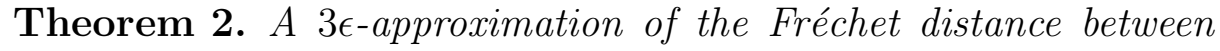
two polygonal curves in a $2 D$ weighted subdivision $R$ can be computed in $O\left(C(R)^{2} \frac{p q}{\epsilon^{2}}\left(\log ^{4} \frac{1}{\epsilon}\right) \bar{q}+\frac{(g+1) n^{2}}{\epsilon^{2 / 3} \bar{q}} \log \frac{n}{\epsilon} \log ^{4} \frac{1}{\epsilon}\right)$ time, where $\bar{q}$ is a query time parameter, $g$ is the genus of the graph constructed by the discretization scheme, $C(R)$ is a constant associated with geometry of $R$, and $n$ is the number of vertices of $R$.

\subsection{Geodesic Fréchet Distance in $\mathcal{R}^{3}$ with Obstacles}

In this subsection, we briefly discuss the geodesic Fréchet distance problem in 1 or $\infty$ weighted regions in $\mathcal{R}^{3}$ (that is, among obstacles in $\mathcal{R}^{3}$ ). Let $R$ be a weighted subdivision in $\mathcal{R}^{3}$ with a total of $n$ vertices. The weight of each region $R_{i} \in R$ is either 1 or $\infty$. Given two polygonal curves $P$ and $Q$ in $R$, we want to find the Fréchet 
distance between $P$ and $Q$, where the distance between two points in $R$ is defined as the length of the geodesic path between those points, i.e. the length of the shortest obstacle-avoiding path.

We set $r(v)=\epsilon B$ and add additional vertices on $P$ and $Q$ as described in Section 4.1. Let $P^{\prime}$ and $Q^{\prime}$ be the new curves.

Lemma 9. The discrete Fréchet distance between $P^{\prime}$ and $Q^{\prime}$ gives an $\epsilon$-approximation of the Fréchet distance between $P$ and $Q$, i.e. $(1-\epsilon) \delta_{F}(P, Q) \leq \delta_{d F}\left(P^{\prime}, Q^{\prime}\right) \leq(1+\epsilon) \delta_{F}(P, Q)$.

Proof. Similar to the proof of Lemma 8.

Let $\delta_{d F}^{\prime}\left(P^{\prime}, Q^{\prime}\right)$ be the discrete Fréchet distance computed by a shortest path approximation algorithm, which gives an $\epsilon$-approximation of the shortest path. We have

$$
\left|\delta_{d F}^{\prime}\left(P^{\prime}, Q^{\prime}\right)-\delta_{F}(P, Q)\right| \leq 3 \epsilon \delta_{F}(P, Q) .
$$

$\delta_{d F}^{\prime}\left(P^{\prime}, Q^{\prime}\right)$ can be computed in $O\left(C(R)^{2} p q\left(1 / \epsilon^{2}\right) \log ^{4}(1 / \epsilon) T(n, \epsilon)\right.$ time, where $C(R)$ is a constant depending on the geometry of the problem and $T(n, \epsilon)$ is the time to approximate the shortest path between two points in $R$. For example, we can use the approximation algorithm given by Clarkson [12], which takes $O\left(n^{2} \lambda(n) \log (n / \epsilon) / \epsilon^{4}+\right.$ $\left.n^{2} \log (n \gamma) \log (n \log \gamma)\right)$ time, where $\gamma$ is the ratio of the length of the longest obstacle edge to the Euclidean distance between the two points, and $\lambda(n)$ is a very slowly-growing function related to the inverse of the Ackermann's function. Thus, our algorithm takes $O\left(C(R)^{2} p q\left(1 / \epsilon^{2}\right) \log ^{4}(1 / \epsilon)\left(n^{2} \lambda(n) \log (n / \epsilon) / \epsilon^{4}+n^{2} \log (n \gamma) \log (n \log \gamma)\right)\right)$ time.

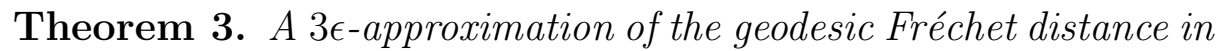
$\mathcal{R}^{3}$ with Obstacles can be computed in $O\left(C(R)^{2} p q\left(1 / \epsilon^{2}\right) \log ^{4}(1 / \epsilon)\left(n^{2} \lambda(n)\right.\right.$ $\left.\left.\log (n / \epsilon) / \epsilon^{4}+n^{2} \log (n \gamma) \log (n \log \gamma)\right)\right)$ time, where $\gamma$ is the ratio of the length of the longest obstacle edge to the Euclidean distance between the two points, and $\lambda(n)$ is a very slowly-growing function related to the inverse of the Ackermann's function.

\section{Conclusion}

In this paper, we discussed three versions of the Fréchet distance problem in weighted regions and presented an approximation algorithm for each version. First, we discussed the non-geodesic Fréchet 
distance problem in planar weighted regions. We showed that we can approximate the Fréchet distance by using a parameter space, $D$, where each leash is associated with a point in $D$, and constructing a discrete graph $G$ from $D$. We then discussed two geodesic Fréchet distance problems, in planar weighted regions and in 1 or $\infty$ weighted regions in $\mathcal{R}^{3}$, and showed that in both cases, by adding additional vertices to the polygonal curves, the discrete Fréchet distance can be used to approximate the continuous Fréchet distance.

\section{References}

1. L. Aleksandrov, H.N. Djidjev, H. Guo, A. Maheshwari, D. Nussbaum and J. Sack. Approximate shortest path queries on weighted polyhedral surfaces. LNCS, 4162:98109, 2006.

2. L. Aleksandrov, A. A. Maheshwari, and J.R. Sack. Approximation algorithms for geometric shortest path problems. In Proc. 32nd Annual ACM Symposium on Theory of Computing, pp. 286-295, 2000.

3. L. Aleksandrov, A. Maheshwari and J. Sack. Determining approximate shortest paths on weighted polyhedral surfaces. Journal of the ACM, 52(1):25-53, 2005.

4. H. Alt and M. Godau. Computation the Fréchet distance between two polygonal curves. International Journal of Computational Geometry and Applications, 5:75-91, 1995.

5. N.M. Amato, M.T. Goodrich, and E.A. Ramos. Computing the arrangement of curve segments: Divide-and-conquer algorithms via sampling. In Proc. 11th Annual CAM-SIAM Symposium on Discrete Algorithms, pp. 705-706, 2000.

6. K. Buchin, M. Buchin and C. Wenk. Computing the Fréchet distance between simple polygons in polynomial time. In Proc. 22rd Symposium on Computational Geometry, pp. 80-87, 2006.

7. K. Buchin, M. Buchin, and Y. Wang. Exact partial curve matching under the Fréchet distance. To appear In Proc. ACM-SIAM Symposium on Discrete Algorithms, 2009.

8. E.W. Chambers, É. Coline De Verdière, J. Erickson, S. Lazard, F. Lazarus and S. Thite. Walking your dog in the woods in polynomial time. In Proc. 24th Annual ACM Symposium on Computational Geometry, pp. 101-109, 2008.

9. D.Z. Chen, O. Daescu, X. Hu, X. Wu and J. Xu. Determining an optimal penetration among weighted regions in two and three dimensions. J. combinat. Optim., 5(1):5979, 2001.

10. S.W. Cheng, H.S. Na, A. Vigneron and Y. Wang. Approximate shortest paths in anisotropic regions. In Proc. 18th annual ACM-SIAM symposium on Discrete algorithms, PP. 766 - 774, 2007.

11. S.W. Cheng, H.S. Na, A. Vigneron and Y. Wang. Querying approximate shortest paths in anisotropic regions. In Proc. 23rd annual symposium on Computational geometry, pp. 84 - 91, 2005.

12. K.L. Clarkson. Approximation algorithms for shortest path motion planning. In Proc. 19th Annual ACM Symposium on Theory of Computing, pp.56-65, 1987

13. G. E. Collins. Quantifier elimination for real closed fields by cylindrical algebraic decomposition. Lecture Notes In Computer Science, 33:134-183, 1975. 
14. A.F. Cook IV and C. Wenk. Geodesic Fréchet distance inside a simple polygon. In Proc. 25th International Symposium on Theoretical Aspects of Computer Science, pp. 193-204, 2008.

15. T. Eiter and H. Mannila. Computing discrete Fréchet distance. Technical Report $C D-T R$ 94/64, Information Systems Department, Technical University of Vienna, 1994.

16. M. Fréchet. Sur quelques points du calcul fonctionnel. Rendiconti del Circolo Mathematico di Palermo, 22:1-74, 1906.

17. J. D. J. Ingle and S. R. Crouch. Spectrochemical Analysis. Prentice Hall, New Jersey, 1988.

18. A. Maheshwari and J. Yi. On computing Fréchet distance of two paths on a convex polyhedron. In Proc. 21st European Workshop on computational Geometry, pp. 4144, 2005.

19. J. S. B. Mitchell and C. H. Papadimitriou. The weighted region problem: Finding shortest paths through a weighted planer subdivision. Journal of the ACM, 38(1):1873, 1991.

20. G. Rote. Computing the Fréchet distance between piecewise smooth curves. Technical Report, ECGTR-241108-01, 2005.

21. Z. Sun and J. H. Reif. On finding approximate optimal path in weighted regions. Journal of Algorithms, 58(1):1-32, 2006. 